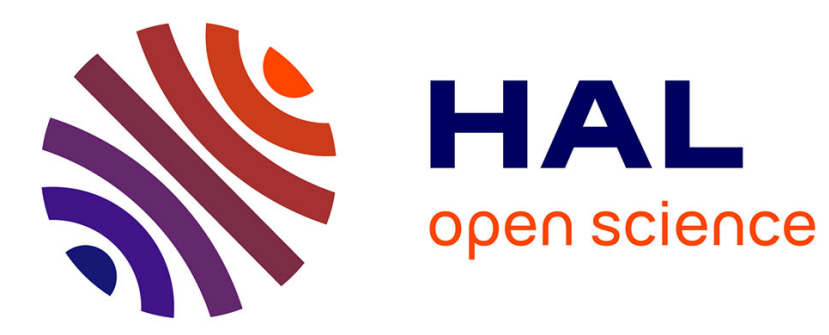

\title{
Sequential adaptive sampling algorithm to reduce the near field measurement time
}

Sébastien Serpaud, Alexandre Boyer, Sonia Ben Dhia

\section{To cite this version:}

Sébastien Serpaud, Alexandre Boyer, Sonia Ben Dhia. Sequential adaptive sampling algorithm to reduce the near field measurement time. International Symposium on Electromagnetic Compatibility (EMC Europe 2019), Sep 2019, Barcelona, Spain. hal-02319466

\section{HAL Id: hal-02319466 https://hal.laas.fr/hal-02319466}

Submitted on 18 Oct 2019

HAL is a multi-disciplinary open access archive for the deposit and dissemination of scientific research documents, whether they are published or not. The documents may come from teaching and research institutions in France or abroad, or from public or private research centers.
L'archive ouverte pluridisciplinaire HAL, est destinée au dépôt et à la diffusion de documents scientifiques de niveau recherche, publiés ou non, émanant des établissements d'enseignement et de recherche français ou étrangers, des laboratoires publics ou privés. 


\section{Sequential adaptive sampling algorithm to reduce the near field measurement time}

\author{
Sébastien Serpaud \\ IRT Saint-Exupéry \\ Toulouse, France \\ sebastien.serpaud@irt-saintexupery.com
}

\author{
Alexandre Boyer \\ CNRS, LAAS, Univ. de Toulouse, INSA, \\ Toulouse, France \\ alexandre.boyer@laas.fr
}

\author{
Sonia Ben Dhia \\ CNRS, LAAS, Univ. de Toulouse, INSA, \\ Toulouse, France \\ sonia.bendhia@insa-toulouse.fr
}

\begin{abstract}
This paper presents a sequential adaptive sampling algorithm in order to reduce measurement time of near-field scan. The originality of this approach is to use a deterministic mesh swept to a sequential progressive adaptive algorithm that defines whether a point must be captured or not. All the parameters of the proposed algorithm is set according near-field characteristics and the measurement setup. This approach is validated on two case studies.
\end{abstract}

Keywords - EMC, NFS, Near Field Scanning, measurement, emission, adaptive sampling

\section{INTRODUCTION}

With the current industrial economic constraints, it is necessary to evaluate electromagnetic behavior of an electronic device as early as possible in development process. If the simulation tools fail to predict effectively the electromagnetic compatibility (EMC) of equipment, the EMC engineer must have efficient investigation tools based on measurements. Since many years, the near-field scanning (NFS) approach applied on electronic device is developed. The NFS performances increase industrial's interest for this EMC investigation measurement method. Limited to a robot moving a probe, the cost of NFS test bench is not expensive. The measurement is done above an electronic device. It is not strongly influenced by the set-up of equipment which ensures a high reproducibility of measurement $(<1 \mathrm{~dB})$.

Despite these qualities, the first limitation of the NFS measurement method backed-up by the industrial is the measurement time. The return of 10 years of experience on the near-field measurement in EMC expertise context, shows that the measurement time range often between $15 \mathrm{~min}$ to 5 hours. Note that this measurement time is rather defined by industrial context that by the need to characterize a complete definition of field radiation of device under test (DUT). In a debug context, capturing only one field component $(\mathrm{Hz})$ around a particular frequency is sufficient to investigate root cause of the EMC issue. However, in other conditions, characterizing a complex industrial electronic devices may require higher measurement time. For example, if a $200 \times 150 \mathrm{~mm}$ aeronautic calculator is scanned with $1 \mathrm{~mm}$ sampling step, if it takes 3 seconds to capture one point in the selected bandwidth of frequency $\quad(30 \mathrm{MHz}-200 \mathrm{MHz} / 200 \mathrm{MHz}-3 \mathrm{GHz}$ with $\mathrm{RBW}=9 \mathrm{KHz} / 120 \mathrm{KHz}$ ), the measurement time for 2 magnetic field components is equal to 50hours.

In spite of this serious limitation, only two papers [1] [2] address this topic. According to the approaches developed in these both papers, we proposed an algorithm to reduce the measurement time. The orientation retained in this paper is to reduce the number of measurement points by capturing only points which bring the most information. This requirement opens the two following question: What are the criterion that defines the most relevant information? How is the surface (or volume in $\mathbb{R}^{3}$ ) above the electronic device mapped to capture only this relevant information? This problem could be solve by sequential process as defined in following part parts. After a brief review of the principles of the sequential adaptive spatial sampling process in section II, the proposed algorithm is described in section III. In section IV and V, validation cases are presented which highlight the performances in terms of reduction of sampling point number and measurement error compared to a full scan.

\section{PRINCIPLES OF SEQUENTIAL AdAPTIVE SPATIAL SAMPLING}

Let $\Omega \subset \mathbb{R}^{2}$ the space domain of measurement $(\Omega$ is often defined as planar surface above DUT but it could be defined in $\mathbb{R}^{3}$ ), ' $\mathrm{N}$ ' is the initial number of spatial sampling points and $P_{N}=\left\{p_{i}=\left(x_{i}, y_{i}, z_{i}\right), p_{i} \in \Omega\right\}_{i=1}^{N}$ the initial set of probe positions. The near field radiated by the DUT could be represented by the regionalized variable $F$ defined on P. Note that $F \subset \mathbb{R}$ for magnitude field measurement, $F \subset \mathbb{C}$ for vector measurement and $F \subset \mathbb{R}^{\mathrm{n}} / \mathbb{C}^{\mathrm{n}}$ for multi-frequency measurement. The set of near field values is defined as $\left\{F\left(P_{N}\right)\right\}$ where $F\left(p_{i}\right)$ represents an achievement of $F$ at the point $p_{i}$.

The objective of sequential adaptive sampling is, from initial knowledge of $F$ at some position, to adapt step after step the spatial meshing of $F$ from its own characteristics evaluated at the beginning of each step. At the end, mesh is refined where $F$ evolves quickly and the mesh is released elsewhere.

The initial phase consists in covering the surface with measurement at an arbitrary number of points. The objective is to split $\Omega$ by an optimal spatial cover (without a priori knowledge). As seen in Fig. 1, several approaches [3] could be used to cover $\Omega$.

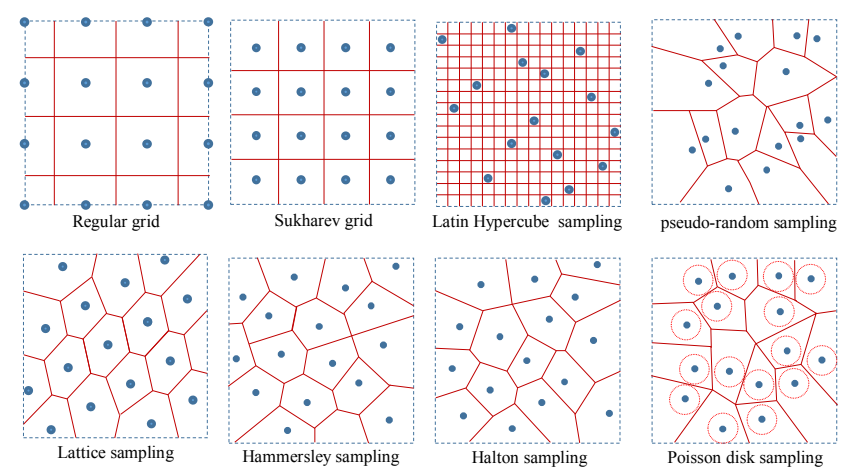

Fig. 1. 16-points Sampling methods

Given a sparse set of data $F\left(P_{N}\right)$ brought by initial step, we can start the sequential adaptive sampling. This iterative process analyzes $\left\{F\left(p_{i}\right)\right\}_{i=1}^{M-N}$ to define where it is necessary to capture the field at a new spatial point to improve the knowledge on $F$, where $\mathrm{M}$ is the total number of sampling point capture at the end of process. 
Each iteration is split in two steps. In the first step, the best candidate position $p_{e}$ to capture the new value of $F$ is looked for. As defined in [1], the Voronoi tessellation approach could be used to identify low sampling region on the space domain. This criterion is only defined from spatial characteristics.

The second step is to assess whether or not adding $F\left(p_{e}\right)$ could significantly improve the knowledge about $F$. A first approach is the analysis of the gradient of $F: \nabla|F(P)|$. The fast change of $|F(P)|$ indicates relevant information of $F$. The gradient of $F$ can be computed from all the set of near field values. But it may be wise to select only the nearest neighbor candidates contained in the radius $\mathrm{d}$ around $p_{e}$ :

$$
\left\{F\left(p_{v}\right),\left\|p_{e}-p_{v}\right\|<d, p_{v} \in p_{i}\right\}_{v=1}^{V}
$$

One can estimate that the value at far distance is very little correlated with the value at the point of interest. This criterion is only defined from the characteristics of the near field measurements (here the magnitude of field). At the end, a final criterion defines if iterations must be stopped and so if the definition of $F$ by $F_{M}=\left\{F\left(p_{i}\right)\right\}_{i=1}^{M}$ is optimal. All not captured points are interpolated from $F_{M}$ to complete full grid.

\section{PROPOSED SEQUENTIAL ADAPTIVE SAMPLING ALGORITHM}

Developing an adaptive algorithm raises several questions to ensure a convergence toward the best solution about the spatial exploration of a function, which is not necessarily well known. As describe in §II, several parameters influence the performance of algorithm to find an optimal spatial sampling.

\section{A. Conditions for collecting the initial dataset}

As all optimization process, the initial set of data $P_{N}=\left\{p_{i}, F\left(p_{i}\right)\right\}_{i=1}^{N}$ could have a serious impact on the optimality of the final solution. The choice of mapping type (and number of initial point) can orient the solution. For example, if the dispersion between samples is high, it is possible to lose relevant information on $F$. Without this information the adaptive algorithm may converge to a bad solution. On the other hand, a dispersion target that is too small could induce a large number of initial samples and therefore a limited reduction objective.

It is very simple and inexpensive to sweep a regular mesh of a surface. Note that we assume here the limitation due to a null dispersion of distance between each point. $\Omega$ is defined to frame the activity of the DUT. So it is reasonable to think that sampling the bound of $\Omega$ does not carry of lot of information on $F$. Adding to a short calculation time, the Sukharev grid combines the minimization of inter-point distance dispersion and the lack of sample point on the border of $\Omega$. The Sukharev grid sampling is used in this algorithm to capture initial set of data.

The number of initial sampling points is an important parameter. A low number of points may miss out relevant information about $\mathrm{Z}$. A high number of initial points conflicts the objective of reducing measurement time. So the number of points of initial dataset must be compute according to the spatial characteristics of near-field. Let be $W$ the sampling rate of initial step of data. This parameter could be optimized following the knowledge on $F$ characteristics. In near field measurement, we are often interesting to capture information above the current flowing in electronic device, which is related to the magnetic near-field. That's why one, two or the three magnetic near-field components are usually measured (using $\mathrm{H}_{\mathrm{xy}}$ or/and $\mathrm{H}_{\mathrm{z}}$ magnetic probes). From the spatial distribution of each magnetic near-field component, it is possible to extract some relevant characteristics about $F$. For example, a magnetic field maximum appears as a main lobe above a thin wire crossed by a current. The initial spatial sampling rate could be defined in order to ensure that a least two measurement points are placed on this lobe. Let $\mathrm{W}_{\mathrm{Hz}(\mathrm{xdB})}$ and $\mathrm{W}_{\mathrm{Hy}(\mathrm{xdB})}$ the width of this lobe according to the attenuation of $\mathrm{x} \mathrm{dB}$ compared to the maximum value of magnetic field as defined in the Fig. 2. Both $\mathrm{W}_{\mathrm{Hz}(\mathrm{xdB})}$ and $\mathrm{W}_{\mathrm{Hy}(\mathrm{xdB})}$ parameters could be extract from the equation of radiated electric dipole (with or without ground plan). Note that the parameters $\mathrm{W}_{\mathrm{Hz}(\mathrm{xdB})}$ and $\mathrm{W}_{\mathrm{Hy}(\mathrm{xdB})}$ are not impacted by the frequency of measurement. With hypothesis that the width of trace $\mathrm{W}_{\text {trace }}$ is lower than the height of measurement $\mathrm{H}_{\text {meas }}$, one can extract the relation between $\mathrm{W}$ (for a specific $\mathrm{x}$ attenuation) and $\mathrm{H}_{\text {meas }}$ as presented in Fig. 3.

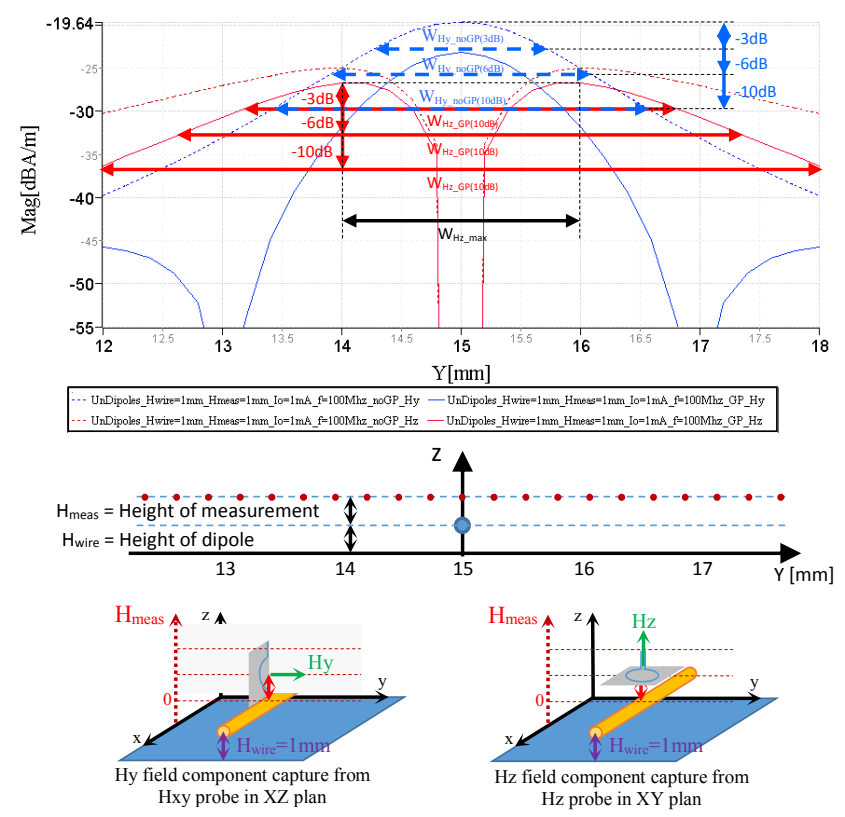

Fig. 2. Spatial distribution of $\mathrm{Hy}$ and $\mathrm{Hz}$ magnetic field above a wire $\left(\mathrm{W}_{\text {trace }} \approx 0\right.$ ) with $1 \mathrm{~mA}$ current of exitation at $100 \mathrm{MHz}$, short dash without ground plan, full line at $\mathrm{H}_{\text {wire }}=1 \mathrm{~mm}$ of a ground plane

\begin{tabular}{|c|c|c|c|}
\hline & Attenuation & $\begin{array}{l}\text { Extraction from electric dipole } \\
\text { equation - Without GP }\end{array}$ & $\begin{array}{l}\text { Criterion } \\
\text { retained }\end{array}$ \\
\hline \multirow{3}{*}{$W_{H y}$} & $-3 \mathrm{~dB}$ & $H_{\text {meas }}$ & \multirow[b]{3}{*}{$\leq 2 . \mathrm{H}_{\text {meas }}$} \\
\hline & $-6 \mathrm{~dB}$ & $1,53 \cdot H_{\text {meas }}$ & \\
\hline & $-10 \mathrm{~dB}$ & $2,15 \cdot H_{\text {meas }}$ & \\
\hline \multirow{4}{*}{$W_{H z}$} & $-3 \mathrm{~dB}$ & $1,66 . H_{\text {meas }}$ & \multirow[b]{3}{*}{$\leq 3 . \mathrm{H}_{\text {meas }}$} \\
\hline & $-6 \mathrm{~dB}$ & $2,14 . H_{\text {meas }}$ & \\
\hline & $-10 \mathrm{~dB}$ & $2,83 . H_{\text {meas }}$ & \\
\hline & Delta Max & $\sqrt{2} \cdot H_{\text {meas }}$ & $\leq 2 . \mathrm{H}_{\text {meas }}$ \\
\hline
\end{tabular}

Fig. 3. Relationship between $\mathrm{W}$ parameters and $\mathrm{H}_{\text {meas }}$ as a function of a specific attenuation and the minimum mesh for two magnetic field components.

From this information, the minimum sampling rate criterion $\mathrm{W}$ for the initial set of data could be defined for selected magnetic field component as presented by the following equation:

$$
\begin{aligned}
& W_{\text {Hxy }} \leq 2 . H_{\text {meas }} \\
& W_{H_{z_{-}} \text {std }} \leq 3 . H_{\text {meas }} \\
& W_{H_{z_{-} \text {fine }}} \leq 2 . H_{\text {meas }}
\end{aligned}
$$


With $\Omega \subset \mathbb{R}^{2}$, one can bound $\Omega$ in the planar surface by $\left\{\mathrm{A}_{\mathrm{x}}, \mathrm{A}_{\mathrm{y}}\right\}$ which define the width of $\Omega$ respectively in $\mathrm{x}$ and $\mathrm{y}$ axes. So the number of points $\mathrm{N}$ collected on the initial process is defined by the following expression:

$$
N=\left\lfloor\frac{A_{x}}{W / d}+1\right\rfloor \cdot\left\lfloor\frac{A_{y}}{W / d}+1\right\rfloor
$$

This equation shows that in the case where the size of $\Omega$ is close to $W$, the number of point could be low. So it is necessary to add a new constraint on $W$ to ensure a minimum number of point $N$ to start the algorithm. Note: in this case, the problem may be poorly defined and it can be necessary to increase the size of $\Omega$ to improve $\mathrm{N}$.

\section{B. Progressive sequential meshing}

The method described in section II proposes to look for an area (around a point) where the mesh can be improved according some criteria from the current mesh. This approach could be time consuming in complex mesh. We proposed here another approach based on progressive sequential meshing as defined in Fig. 4. With this method it is not necessary to use a complex algorithm to find location where it is necessary to refine mesh. The total mesh is built at the beginning.

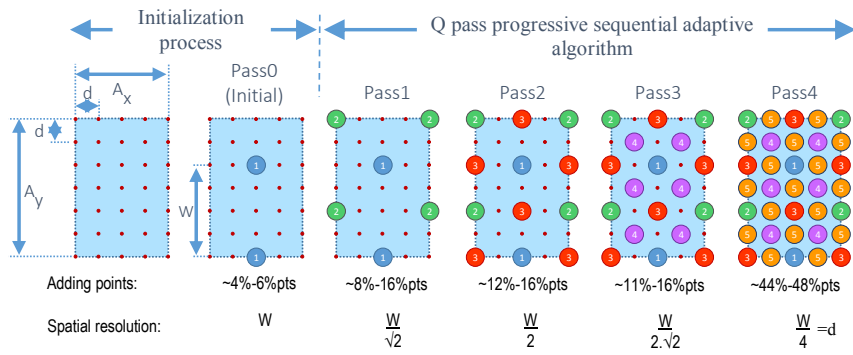

Fig. 4. Example of progressive sequential meshing where $\Omega=\{\mathrm{Ax}, \mathrm{Ay}\}$ is covered on 5 pass according to the parameters : $d$ and $\mathrm{W}$

The value $d$ of the final resolution could be defined from the design and by the measurement probe resolution. When the near-field emission produced by a power electronic device (motor controller for example) or a silicon of microcontroller is characterized, the spatial distribution of near-field and thus the required spatial resolution of the NFS are not the same. This is why it is necessary to select a probe with resolution adapted to the design. In this case, the final resolution of meshing is linked to the probe resolution. It is commonly accepted that a magnetic probe resolution is equal to the radius of loop. So $d$ value will be set to the radius of magnetic probe used for the measurement.

$$
d=r_{\text {loop }}
$$

A progressive spatial sweep is done on a number of sequential pass $Q$. $Q$ could be defined from $d$ and $W$ parameters according to the following equation where $\mathrm{k}$ is the first integer that validate $W / d \geq 2^{k}$ :

$$
Q=2 \cdot k+1
$$

On each pass, a reduced set of point $P q_{\max }=\left\{p_{q i}, q=1 \ldots Q\right\}_{i=1}^{N q_{\max }}$ is defined to progressively cover $\Omega$ at the final resolution $d$. Let $P q$ the set of $N q$ sampling points swept on each pass where $N q \leq N q_{\max }$. Note that both the evaluated number of points and spatial resolution are progressively increased pass after pass as presented in Fig. 4.

\section{Adapative meshing criterion}

During the sweep of $P q_{\max }$ points, the selection criterion is built to define whether or not the value $F(p q i)$ must be captured. One looking for to collect the final set $F_{M}=\left\{F\left(p_{i}\right), p i \in \Omega\right\}_{i=1}^{M}$ where $\quad M=N+\sum_{q=1}^{Q} N_{q}$ define the total number of measurement of $F$ at specific positions $p i$. The objective is to minimize $M$, by ensuring that the final set of $M$ measurements of $F$ allows to build an estimator that minimizes the prediction error of $F$ at any position on $\Omega$. During iteration of $N q_{\max }$ points during the pass $q$, a selection criterion validates whether or not the value $F\left(p_{q i}\right)$ must be measured.

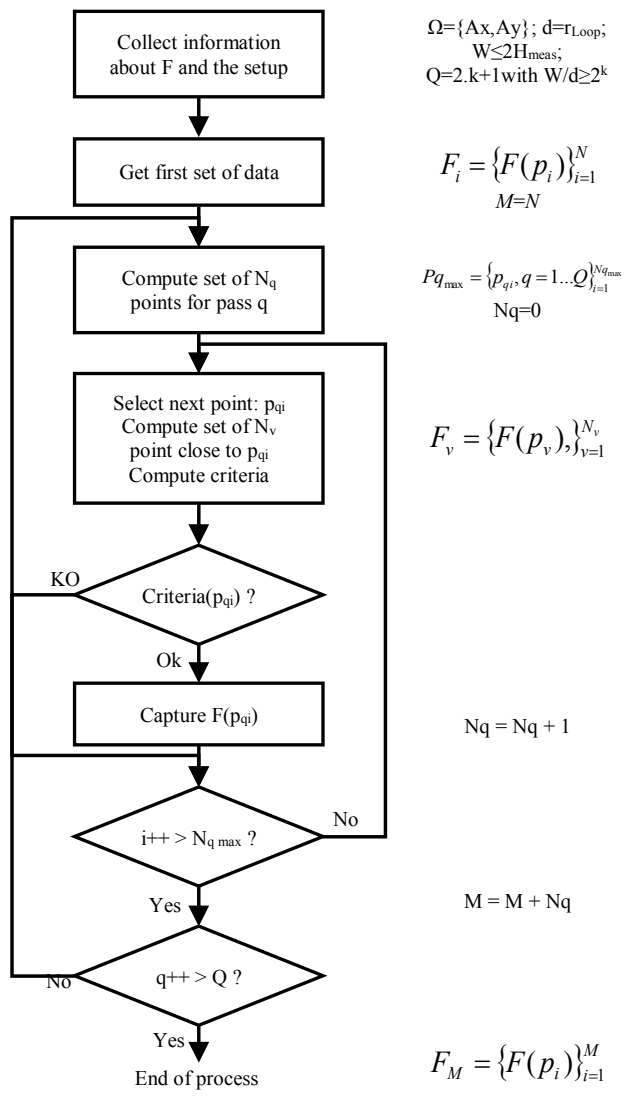

Fig. 5. Diagram of progressive sequential adaptive meshing

The definition of this criterion is the main point of this algorithm. A good sample must reduce the prediction error otherwise the sample is not captured. In others words, this selection criterion defines whether or not a sample at the position pi provides relevant information about $F$.

This criterion is based on the standard deviation principle calculated from the known values of $F_{v}=\left\{F\left(p_{v}\right),\right\}_{v=1}^{N_{v}}$ close to $p_{q i}$. We assume that the noise of measurement is low. It is commonly accepted that the repeatability of near field measurement is good $(<1 \mathrm{~dB})$. According to the definition of the $W$ parameter, the deviation from the mean tolerated was fixed at $10 \mathrm{~dB}$. To increase the effect of the points closest to $p_{q i}$, the mean value of neighbor is weighted by the distance of each point with $p_{q i}$. We thus define the rule that it is necessary to have 2 sampling points for a near field variation of $10 \mathrm{~dB} / \mathrm{W}$. 

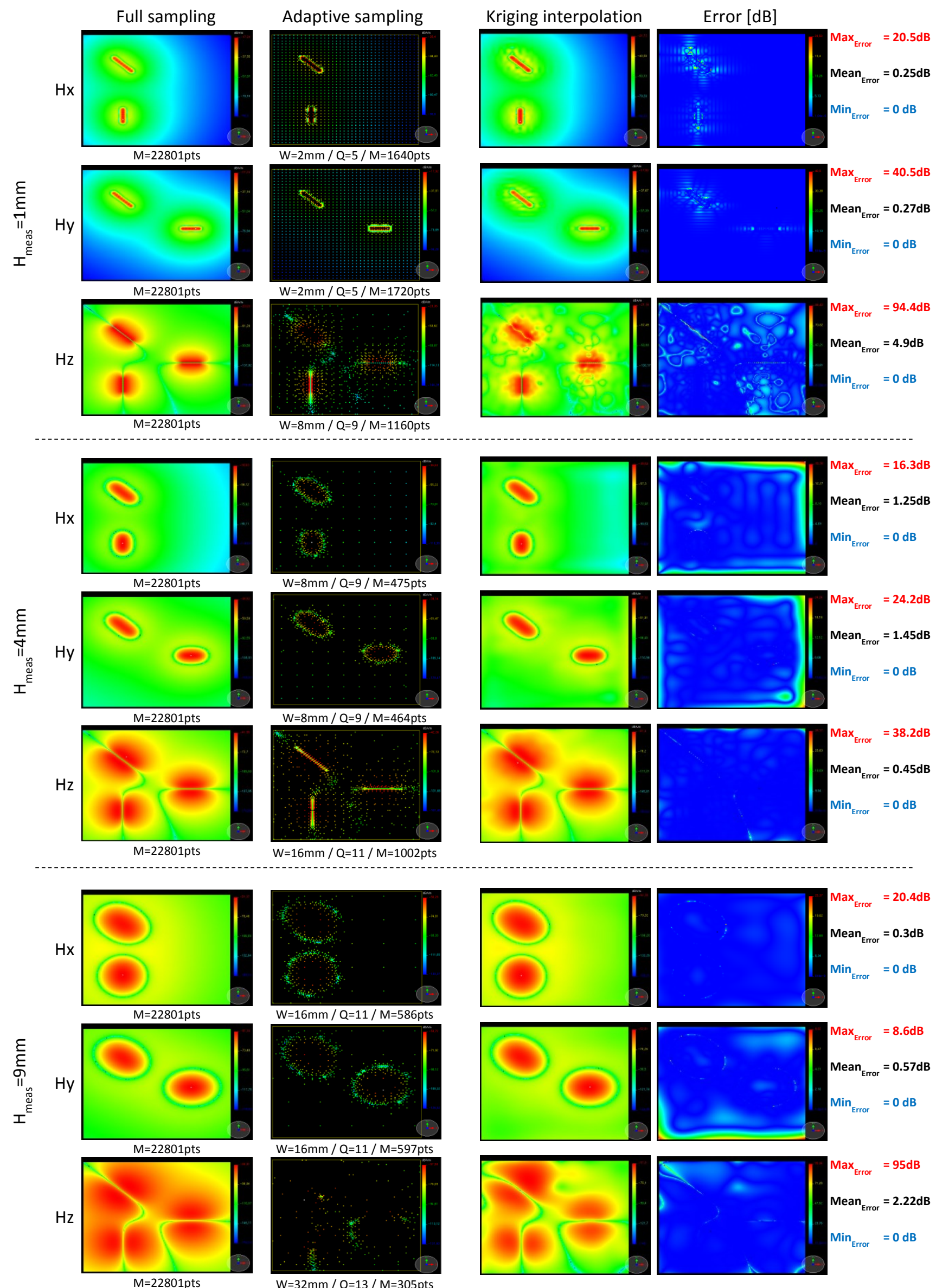

Fig. 6. Comparison between full sampling and optimized sampling from adaptive algorithm for three magnetic field components and three height $(1,4,9 \mathrm{~mm})$ 

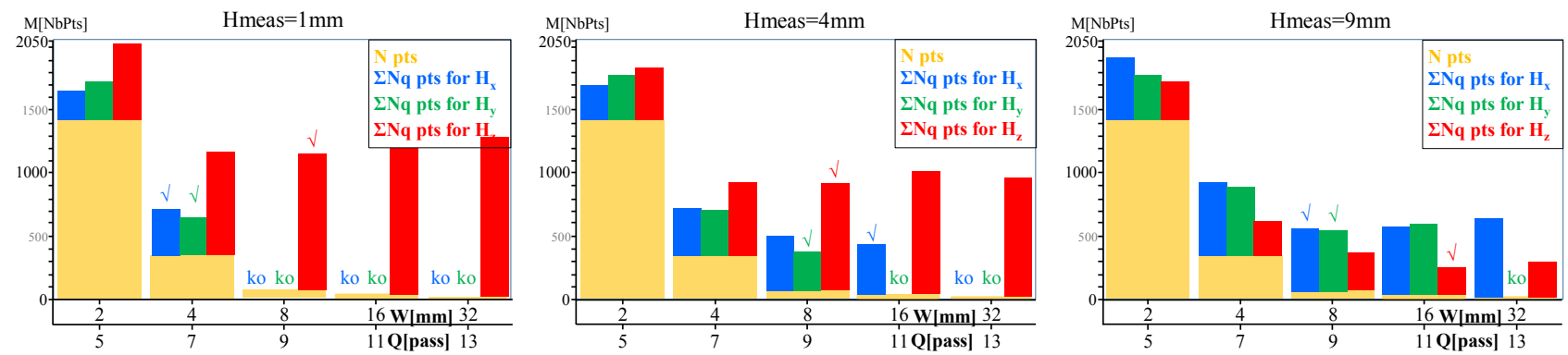

Fig. 7. Number of sampling point $(\mathrm{M}=\mathrm{N}+\Sigma \mathrm{Nq})$ according to magnetic field components and both $\mathrm{W}$ and Height of measurement parameters (Study Case 1)

\section{CASES STUDY}

\section{A. Case study $1:$ simulation of near-field emission on a} virtual PCB including three traces above ground plane

The proposed case study is a $75 \mathrm{~mm} \times 75 \mathrm{~mm}$ printed circuit board (PCB) including three traces with a length of $10 \mathrm{~mm}$ routed in air at $1 \mathrm{~mm}$ above complete ground plane. The three traces are routed with width $=0.05 \mathrm{~mm}$ in three different directions $\left(0^{\circ}, 45^{\circ}\right.$ and $\left.90^{\circ}\right)$ and all are excited by a current source of $1 \mathrm{~mA}$ at $100 \mathrm{MHz}$.

The magnitude of the three magnetic field components is computed at several heights $(1,4$ and $9 \mathrm{~mm})$ above the top of board using analytic equations of electric dipole. We assume a resolution of $\mathrm{d}=0.5 \mathrm{~mm}$. This data is used as input for the adaptive meshing algorithm.

B. Case study 2 : Magnetic near-field measurment done on demonstrator around DSPic microcontrollor that exited bus of six traces at $5 \mathrm{MHz}$.

The proposed case study is a $100 \mathrm{~mm} \times 100 \mathrm{~mm}$ PCB including a DSPic33F microcontroller running at 40MIPS. A bus of six traces is driven by 3,3volts digital signals which switch at $5 \mathrm{MHz}$. A $2 \mathrm{~mm}$ diameter loop is used as $\mathrm{Hz}$ probe to capture the magnetic near field on a surface of $58 \times 79 \mathrm{~mm}$ above $2 \mathrm{~mm}$ of the PCB surface. We assume the resolution of probe $\mathrm{d}$ is equal to $1 \mathrm{~mm}$.
Study case 1:

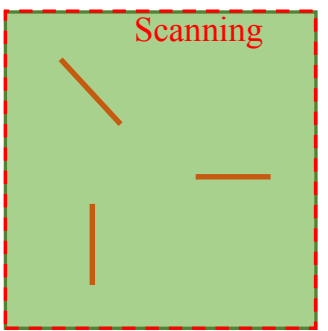

Study case 2:

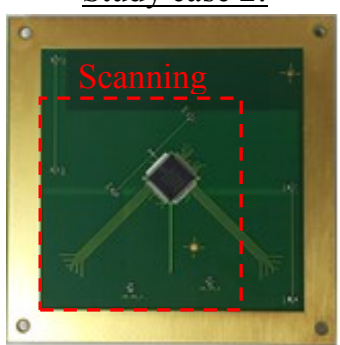

Fig. 8. Study cases 1 : virtual demonstrator including three traces above ground plane

\section{VALIDATION}

The main objective of this work is to reduce the time of the measurement. To reach this goal, we have proposed a progressive sequential adaptive algorithm in order to reduce the number of sampling point while minimizing the error on the reconstruction of $F$ over $\Omega$. After the validation of the definition of $W$, we propose a discussion about these two points.

\section{A. Definition of $W$ from height of measurement}

From equations (2) and (5), it is possible to define $W$ and the number of passes $Q$ according to the measurement height as presented in the next figures.

\begin{tabular}{|c|c|c|c|c|c|}
\hline $\mathbf{H}_{\text {meas }}$ & $\mathbf{W}_{\max }$ & $\mathbf{W}$ & $\mathbf{W} / \mathbf{d}$ & $\mathbf{k}$ & $\mathbf{Q}$ \\
\hline $1 \mathrm{~mm}$ & $2 \mathrm{~mm}$ & $2 \mathrm{~mm}$ & $4 \mathrm{pts}$ & 2 & 5 \\
\hline $4 \mathrm{~mm}$ & $8 \mathrm{~mm}$ & $8 \mathrm{~mm}$ & $16 \mathrm{pts}$ & 4 & 9 \\
\hline $9 \mathrm{~mm}$ & $18 \mathrm{~mm}$ & $16 \mathrm{~mm}$ & $32 \mathrm{pts}$ & 5 & 11 \\
\hline
\end{tabular}

Fig. 9. $Q$ number of pass definition according the heigth of measurement for $\mathrm{Hx}$ or $\mathrm{Hy}$ measurements and $\mathrm{Hz}$ measurement using fine criterion

\begin{tabular}{|c|c|c|c|c|c|}
\hline $\mathbf{H}_{\text {meas }}$ & $\mathbf{W}_{\max }$ & $\mathbf{W}$ & $\mathbf{W} / \mathbf{d}$ & $\mathbf{k}$ & $\mathbf{Q}$ \\
\hline $1 \mathrm{~mm}$ & $3 \mathrm{~mm}$ & $4 \mathrm{pts}$ & $8 \mathrm{pts}$ & 3 & 7 \\
\hline $4 \mathrm{~mm}$ & $12 \mathrm{~mm}$ & $8 \mathrm{pts}$ & $16 \mathrm{pts}$ & 4 & 9 \\
\hline $9 \mathrm{~mm}$ & $27 \mathrm{~mm}$ & $16 \mathrm{pts}$ & $32 \mathrm{pts}$ & 5 & 11 \\
\hline
\end{tabular}

Fig. 10. Q number of pass definition according the heigth of measurement for $\mathrm{Hz}$ measurements (with standard criterion)

Fig. 6 presents the comparison between full sampling at $\mathrm{d}=0.5 \mathrm{~mm}$ and optimized sampling using adaptive algorithm for the three magnetic field components $(\mathrm{Hx}, \mathrm{Hy}$ and $\mathrm{Hz})$ at three heights of measurement $(1,4$ and $9 \mathrm{~mm})$ process on the study case 1 . On Fig. 7, the tip under histogram marks the optimal configuration according to the number of point and the moving time. If we compare the theoretical value of $W / Q$ parameters resume on Fig. 9 and Fig. 10 with $W$ denoting the optimal configuration on Fig. 7, we observe a good agreement. The theoretical value of $W / Q$ almost always defines the best configuration. Note that if $W$ is greater than this theoretical value, a significant loss of information on $F$ (marked by ko) is observed. This result validates the criterion on $W / Q$ parameters defined by equations (2) and (5).

With $\mathrm{Hz}$ magnetic field component measured at $9 \mathrm{~mm}$, we have reached the limit of our theoretical analysis about the performance of this algorithm. Indeed, on nominal conditions, a larger diameter probe would have been used at such height of measurement. A larger probe induce a greater spatial integration effect of the near-field. So $d$ would have been higher. In this case, the result will be better than presented here in terms of measurement point reduction and reconstitution.

The effectiveness of the approach have been tested with success on measurement data from case study 1 as presented in Fig. 11. 


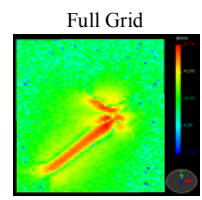

$\mathrm{M}=4720 \mathrm{pts}$

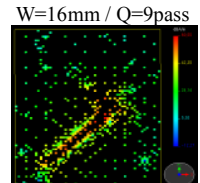

$\mathrm{M}=888 \mathrm{pts}$

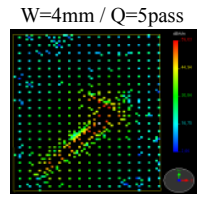

$\mathrm{M}=1539 \mathrm{pts}$

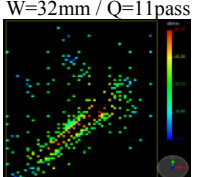

$\mathrm{M}=831 \mathrm{pts}$
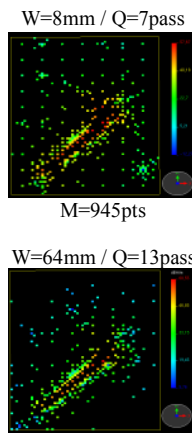

$\mathrm{M}=822 \mathrm{pts}$
Fig. 11. Comparison between full sampling and optimized sampling from adaptive algorithm applied on case study 2 .

\section{B. Interpolation-evaluation of information loss}

The main objective of the progressive adaptive meshing is to reduce the number of sampling point without lost information about $F$. In the previous section, we have validate that the algorithm reduces the number of sampling points. The next step is to confirm that the loss of information caused by the reduction of sampling point remains acceptable.

Kriging interpolator, also known as Gaussian Process regression, is an efficient spatial unbiased estimator with minimum variance. The Scikit library [6] has been used to process adaptive meshing interpolation. Interpolator is initialized with the Matern kernel. Fig. 6 shows the interpolation of all the maps obtained from the adaptive algorithm on case study 1 . One can observe a very good correlation between the interpolated and the full grid maps. An error map shown in the right column of Fig. 6, plots the error in $\mathrm{dB}$ between interpolated map and full grid map. It attests that the level of information loss on $F$ remains low using adaptive meshing algorithm. To confirm this one, an error map, shows on the right column of Fig. 6, was built to display error in $\mathrm{dB}$ between interpolated map and full grid map. An error lower than $5 \mathrm{~dB}$ around high emission area is observed.

Note that the error tends to increase in the boundaries of the map. This effect is due to the Kriging interpolator that is not performing to extrapolate beyond the initial dataset.

\section{Measurement time optimization}

Fig. 12 presents a summary of measurement time for several $W$ values. The durations related to the field acquisition (acquisition of $F_{M}$ dataset), the execution of the algorithm and near-field probe displacement are identified for each value of $W$. To calculate the measurement time, we assume that it takes 1second per point. Moreover, a constant speed of $20 \mathrm{~cm} /$ second is supposed to compute the probe displacement time. Probe acceleration and deceleration phases are not taken into account.

As presented in Fig. 12, the probe displacement time is negligible. This is the benefit of using a regular mesh (Sukharev grid) coupling to the progressive sweep described in Fig. 4. Therefore the distance between two consecutive points is minimized.

In all configurations, the algorithm time is lower than one second $(\sim 350 \mathrm{~ms} \pm 100 \mathrm{~ms})$. The most important contribution to the measurement time is the acquisition time.

A full grid measurement lasts around 6hours and 23 minutes to process the 22801 points of the map. The same measurement using the adaptive algorithm is reduced to 17 minutes, i.e. the measurement time gain is about 22 . On the case study 2 , the time of measurement is reduced to $19 \mathrm{mins}$ with our algorithm compared to 1 hour and 22 mins for the scan with full sampling.

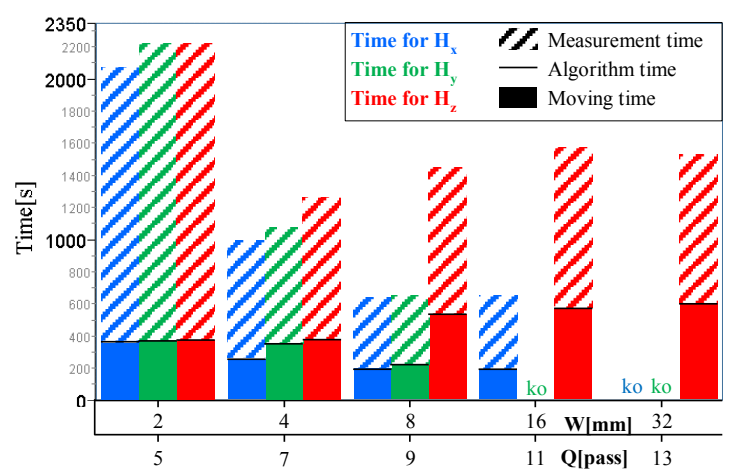

Fig. 12. Time breakdown following $\mathrm{W}$ (or $\mathrm{Q}$ number of pass) for $\mathrm{Hz}$ measurements $\left(\mathrm{H}_{\text {meas }}=4 \mathrm{~mm}\right)$

\section{CONCLUSION}

This paper proposes a sequential adaptive sampling algorithm. We have demonstrated that it is possible to define the parameters of the algorithm according to the characteristics of the spatial distribution of the near- field and measurement configuration. The performance of the algorithm was tested on a simple PCB. The optimal parameters proposed by our method are validated. Our approach provide a gain of a factor of 4 up to 20 on the measurement time, without introducing excessive measurement errors (less than $5 \mathrm{~dB}$ ).

We have proposed a selected criterion based on the characteristic of magnetic near-field magnitude. This criterion has been validated on two case studies. It would be necessary to validate this algorithm on more case studies including on electric near-field measurement. The selected criterion is based on $F \subset \mathbb{R}$. Multi-frequency measurements and vector measurements have not been evaluated. The definition of a specific criterion would surely be necessary. The proposed adaptive algorithm is developed for the near-field emission measurement. But it could be easily extended to the near-field immunity.

\section{ACKNOWLEDGMENT}

This research was supported by the IRT Saint-Exupery foundation (a French Institute of Technology) under the FELINE project. I would especially like to thank Matthias for his help on the Kriging tool.

\section{REFERENCES}

[1] D. Deschrijver, D. Pissoort, T. Dhaene, "Automated near-field scanning algorithm for the EMC analysis of electronic devices," IEEE Transactions on EMC, vol. 54, no. 3, pp. 502-510, June 2012

[2] S. Serpaud, S. Leman, "Technical improvement of the near field measurement : The vector near field scanning", EMC Europe 2013, Brugge, Belgium, September 2-6, 2013

[3] S. M. LaValle, M. S. Branicky, S. R. Lindemann, "On the Relationship between Classical Grid Search and Probabilistic Roadmaps.", The International Journal of Robotics Research, 23(7-8), 673-692 (2004). https://doi.org/10.1177/0278364904045481

[4] A. G. Sukharev. Optimal strategies of the search for an extremum. U.S.S.R. Computational Mathematics and Mathematical Physics, 11(4), 1971. Translated from Russian, Zh. Vychisl. Mat. i Mat. Fiz., 11, 4, 910-924, 1971.

[5] IC-EMC software. http://www.ic-emc.org

[6] The Scikit library https://scikit-learn.org 